\title{
THE USE OF ECHELLE GRATINGS IN ULTRAVIOLET SPACE ASTRONOMY
}

\author{
B. C. BOLAND, W. M. BURTON, B. B. JONES, and N. K. REAY \\ Astrophysics Research Unit, Culham Laboratory, England
}

\begin{abstract}
The echelle diffraction grating can be used to provide high spectral resolution in compact instrumentation which is very suitable for space astronomy applications. Spectrographs in which the high dispersion of the echelle is crossed with that of a concave grating have been flown in 'Skylark' sounding rockets to record the solar spectrum in the wavelength range $1200 \AA-2200 \AA$, and further instrumentation is in preparation for observation of stellar spectra. Efficiency measurements have been made on some echelle replicas used in the instruments, and are correlated with surface conditions on the reflecting facets of the rulings. Results are also presented to demonstrate a spectral resolving power of $\lambda / \Delta \lambda \approx 10^{5}$ over the wavelength range.
\end{abstract}

\section{Introduction}

The work of Harrison (1949) at M.I.T. in developing the ruling of echelle gratings has resulted in a rapid increase in their applications in recent years. In particular they have proved suitable for providing stable compact high resolution spectrographs which can be used in space vehicles for measurements at vacuum ultraviolet wavelengths down to about $1200 \AA$.

In designing spectroscopic instruments for these applications two of the important echelle characteristics to be considered are spectral efficiency and spectral resolution. and the data we present here have been obtained during the preparation of echelle spectrographs for use in sounding rockets to study both solar and stellar spectra.

\section{Efficiency}

Efficiency measurements have been made on four echelles over the wavelength range $900 \AA-4400 \AA$ (Burton and Reay, 1970). The gratings are Bausch and Lomb replicas obtained from two different masters, both having 73.25 grooves $/ \mathrm{mm}$, and a blaze angle of $63^{\circ} 26^{\prime}$. Three of the gratings were coated by the makers with $\mathrm{Al}+\mathrm{MgF}_{2}$, while the other was coated with Al only.

The optical system used to determine the efficiencies is shown in Figure 1. G is the concave grating of a Seya-Namioka monochromator which was used to select strong spectral lines from a source $L$. The exit slit of the monochromator formed the entrance slit of a Czerny-Turner spectrometer with path folding mirrors $M_{1}, M_{2}$, concave mirrors $C_{1}, C_{2}$, and the echelle under test, $E$. The detector $P$, at the exit slit was a sodium salicylated end window photomultiplier, E.M.I. type 9524B. The echelle was mounted on a turntable system which could be operated from outside the vacuum system. Also mounted on the turntable was a plane reference mirror $R$ which was accurately located to place its reflecting surface co-planar with the reflecting facets of the echelle grooves. Measurements of the photomultiplier signal. first with the refer- 
ence mirror positioned in the parallel beam from $C_{1}$, and then with the echelle in the same position, allowed the echelle efficiency to be compared directly with the reflectivity of the plane reference mirror. The reflectivity of the reference mirror as a function of wavelength was determined in a separate series of measurements and enabled the echelle efficiency to be converted to absolute values at a number of selected wavelengths.

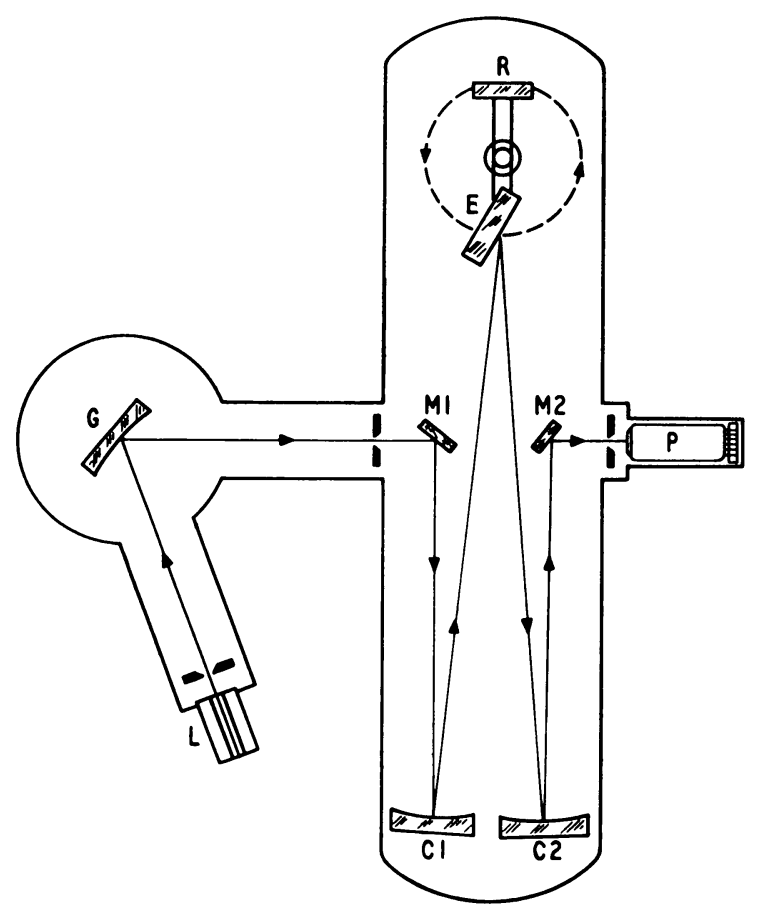

Fig. 1. Diagram of the optical system used to obtain echelle efficiency measurements. $L$, light source; $G$, concave grating in monochromator; $\mathrm{M} 1, \mathrm{M} 2$, plane path-folding mirrors; $C 1, C 2$, concave mirrors; $E$, echelle grating; $R$, reference-mirror; $P$ photomultiplier detector.

The measurements were made on the intense central order and also on the adjacent subsidiary orders of the echelle spectrum, and by fitting the measured intensities in the different orders to the curve for the theoretical diffraction pattern (Schroeder, 1967) it was possible to determine the efficiency which would be obtained if the particular wavelength was located at the blaze peak. This allows the data to be presented on a consistent basis, which is independent of the position of a wavelength within the echelle blaze envelope. The efficiency measurements for the four echelles, normalized in this manner, are shown in Figure 2 together with the reflectivity curve for the reference mirror. The data demonstrate that different replicas from the same master have similar performances, but that two individual master echelles can have quite different characteristics.

The efficiencies of the replicas from the 156 master are almost identical down to a 
wavelength of $1400 \AA$, but below this point $156-\mathrm{A}$, overcoated with $\mathrm{MgF}_{2}$, shows enhanced efficiency compared with 156-B which was coated with $\mathrm{Al}$ only. In the pair of replicas from the other master, one of them, 180-B suffered an accidental contamination with silicone vacuum pump oil and was subsequently cleaned by the manufacturers but was not re-coated. The deterioration in the reflectivity compared with $180-\mathrm{A}$ is evident below $3000 \AA$.

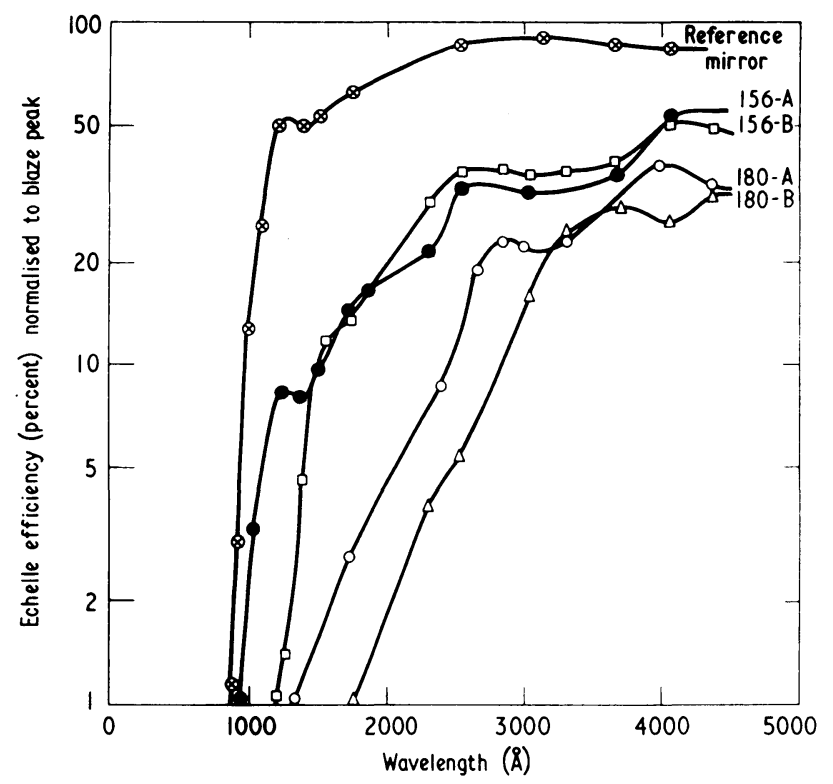

Fig. 2. Measurements of echelle efficiency normalized to blaze peak plotted as a function of wavlength. The upper curve shows the reflectance of the plane reference mirror. The measured points have an estimated accuracy of $3{ }^{\circ} \%$

Photomicrographs have been taken of the groove form on replicas of both masters and are reproduced in Figure 3. The grooves in the 156 replica are seen to be smooth and well defined, indicating good specular reflective properties, whereas the 180 replica exhibits irregular unpolished facets producing scattering of the incident radiation and a loss of efficiency.

\section{Resolution}

Three of the echelle gratings, 156-A, 180-A and 180-B, have been used in a rocket mounted spectrograph designed to record the solar spectrum from $1200 \AA-2200 \AA$. (Boland et al., 1971).

The optical system of the spectrograph is shown in Figure 4. Light from the sun falls initially onto a collector mirror, which is external to the spectrograph. and is then focussed to form an image of the solar disc on the entrance slit. Light passing through the slit is made parallel by the collimator and is directed to the echelle. which is 
mounted with its grooves parallel to the slit, and which is tilted slightly to reflect the beam onto a concave diffraction grating. The rulings of the concave grating are at rights angles to those of the echelle (and to the slit) and the spectrum is formed in a two dimensional array in the focal plane of the grating to be recorded on film. Figure 5 shows a laboratory molecular spectrum covering the range $1200 \AA-2200 \AA$ using a microwave excited source with a filling gas of $\mathrm{CO}+$ air at a pressure of 0.4 Torr.

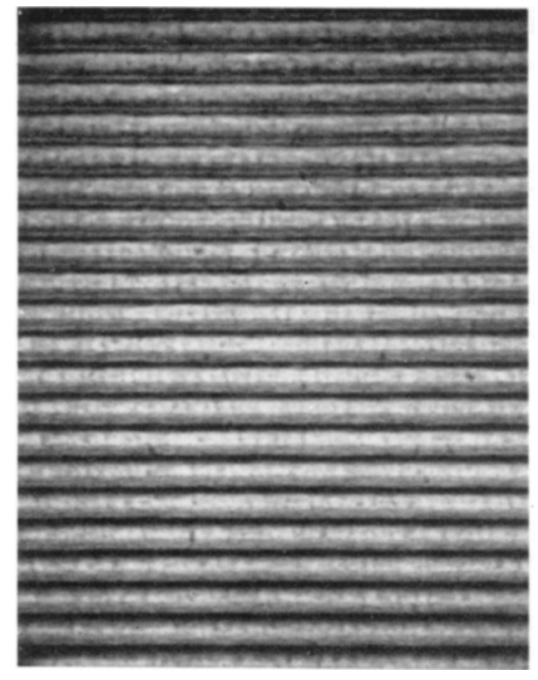

180-A

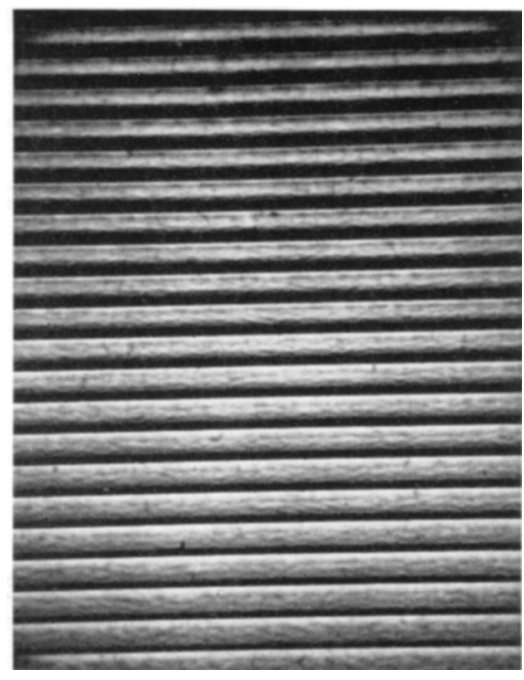

156-B

Fig. 3. Photomicrographs showing the groove form for two replica echelle gratings from different masters.
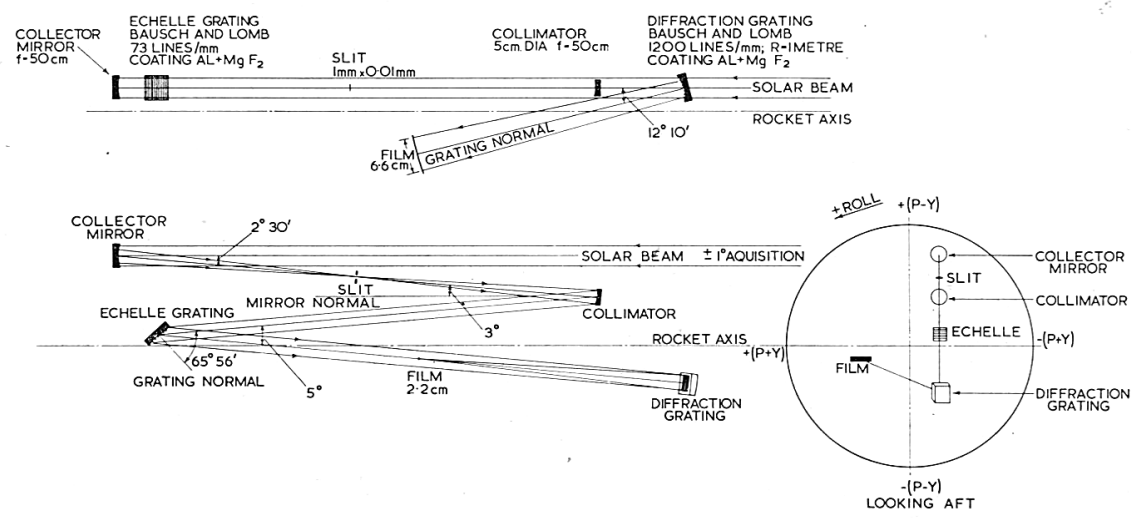

Fig. 4. The optical diagram of the rocket-mounted solar echelle spectrograph. 


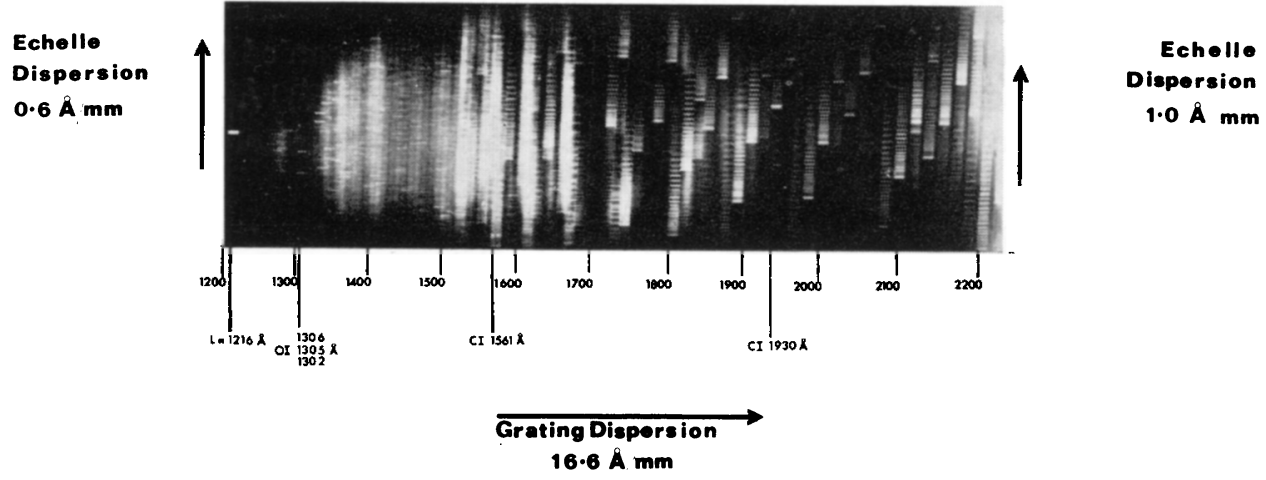

Fig. 5. The spectrum from a laboratory microwave excited source with a filling gas of $\mathrm{CO}+$ air at a pressure of 0.4 Torr. Exposure time 2 min. Kodak 101-01 emulsion.

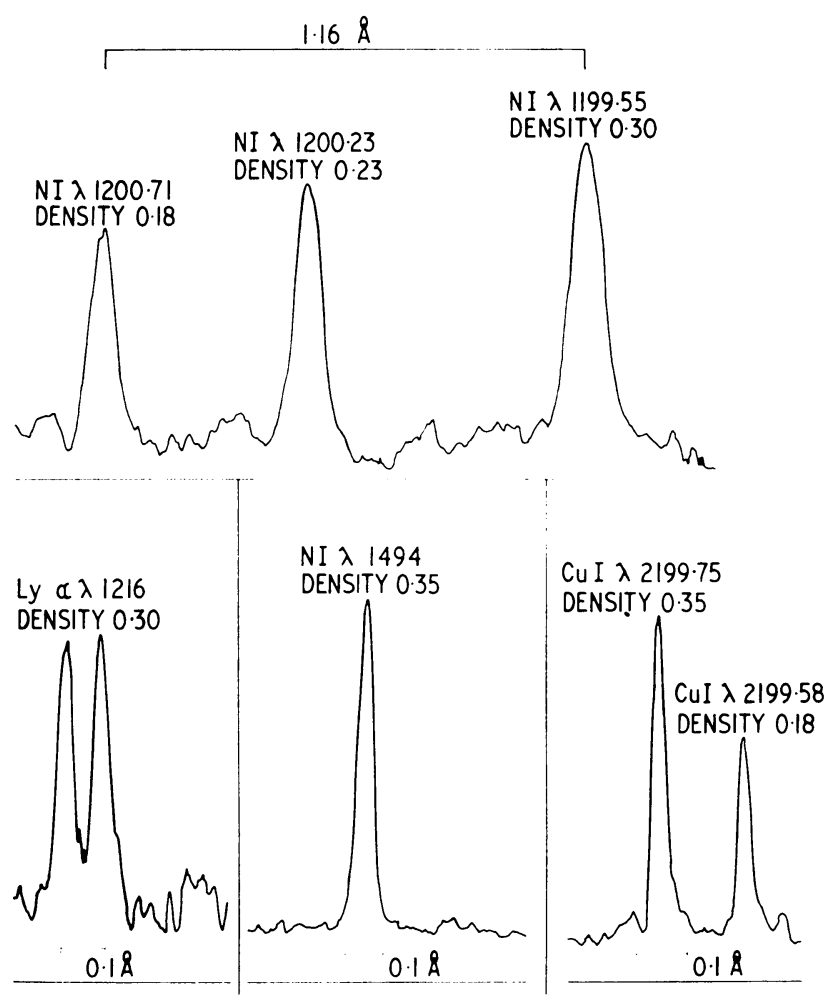

Fig. 6. Densitometer profiles of atomic lines from laboratory sources. The lines are source broadened. 
The resolution of such a system can be influenced by a number of factors including aberrations of the echelle and concave grating, the slit width, the grain size of the photographic emulsion and also thermal effects within the instrument. The theoretical resolution of the echelle for wavelength $\lambda$ is given by Harrison (1949) as

$$
\lambda / \Delta \lambda=2 W \sin \alpha / \lambda
$$

where $W \quad=$ the ruled width of the echelle

and $\alpha \quad=$ the angle of incidence

so that $W \sin \alpha$. = the optical depth

In the present instrument

$$
\begin{aligned}
& \lambda / \Delta \lambda=1.44 .10^{6} \text { at } \lambda 1200 \AA \\
& \lambda / \Delta \lambda=1.16 .10^{6} \text { at } \lambda 1500 \AA
\end{aligned}
$$

and

$$
\lambda / \Delta \lambda=0.87 .10^{6} \text { at } \lambda .2000 \AA
$$
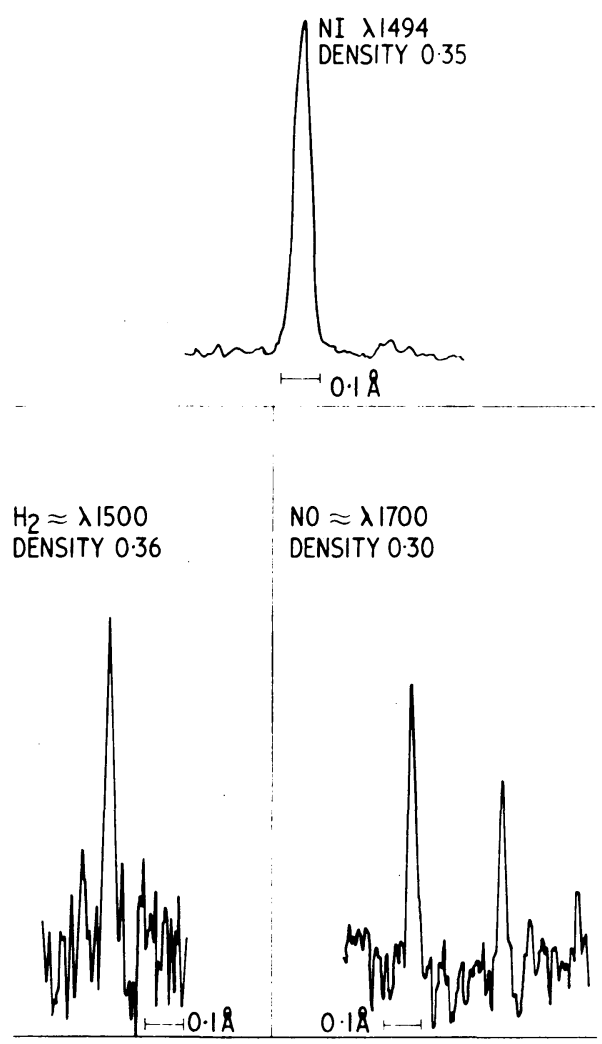

Fig. 7. A broadened atomic line of NI $\lambda .1494 \AA$ compared with narrow molecular lines from the microwave source. The molecular lines have widths similar to the photographic grain fluctuations. 
However, with the photographic emulsions in current use, Kodak-Pathé 'SC' types and Kodak 101-01, the developed grain size is in the region of $0.020 \mathrm{~mm}$, which limits $\lambda / \Delta \lambda$ to $\sim 10^{5}$ over the full wavelength range. The slit width is therefore adjusted to match the emulsion grain size and is operated at the widest setting which does not cause a deterioration in the resolution. Figure 6 shows profiles of selected lines covering the full wavelength range of the instrument. The lines in this figure however are all source-broadened and can only indicate the quality of profile shape that can be achieved. In Figure 7, a broadened line profile near the centre of the range is compared with profiles of lines from the molecular source, where it can be seen that the widths of the molecular lines are similar to the photographic grain fluctuations on the densitometer trace.

Photographic detection of high resolution spectra at these wavelengths usually requires the use of the very fast exposed-grain types of emulsion with the consequent limitations on achievable resolution. For this reason no attempt has been made to use finer grain emulsions to determine whether the groove forms shown in Figure 3 can be correlated with the spectral resolution of individual echelle gratings. The resolution which can be achieved however is sufficient for a wide range of investigations of either emission or absorption line profiles in solar and stellar spectra at these wavelengths. and Figure 8 shows part of a solar spectrum at this resolution which was obtained on a recent rocket flight.

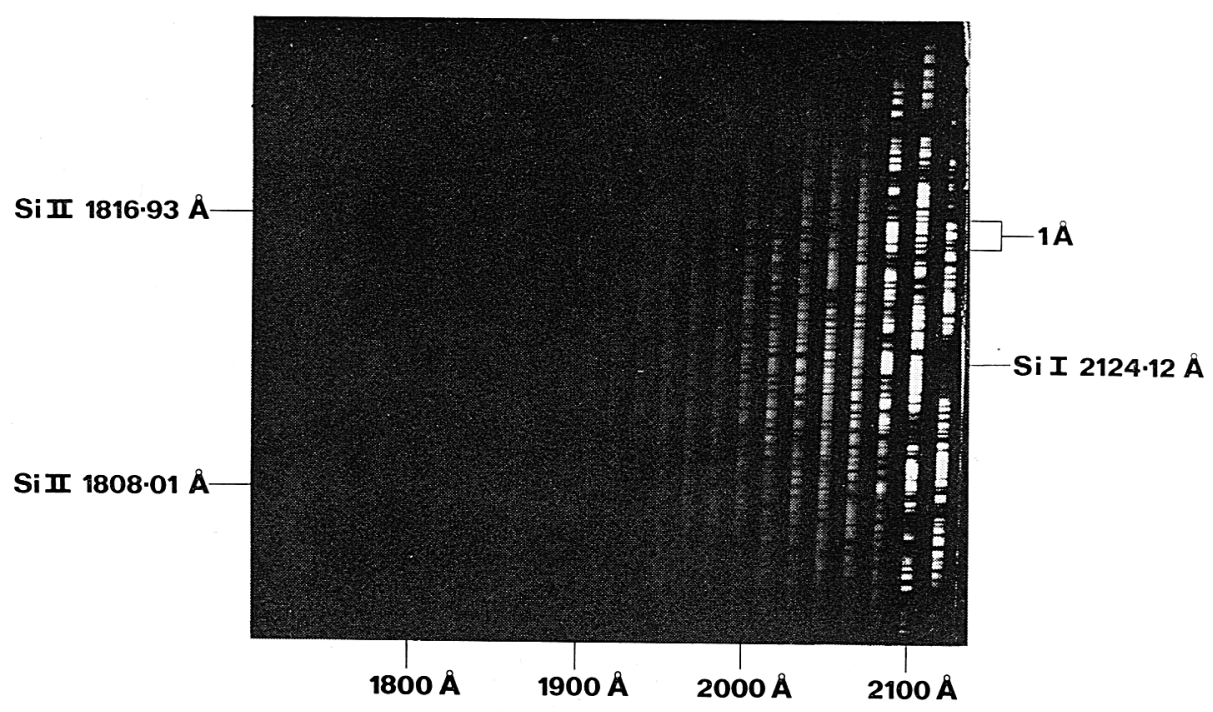

SKYLARK SL803 APRIL 7th 1970

Fig. 8. A high resolution solar spectrum $(\Delta i \approx 0.02 \AA)$ obtained on a recent flight of a Skylark sounding rocket. Exposure time $25 \mathrm{~s}$, altitude $\sim 170 \mathrm{~km}$. Kodak 101-01 emulsion. 


\section{References}

Boland, B. C., Jones, B. B., Wilson, R., Engstrom, S. F. T., and Noci, G.: 1971, Phil. Tran. Roy. Soc. Lond. A270, 29.

Burton, W. M., and Reay, N. K.: 1970, Appl. Opt. 1227.

Harrison, G. R.: 1949, J. Opt. Soc. Am. 39, 522.

Schroeder, D. J.: 1967, Appl. Opt. 6, 1976.

\section{DISCUSSION}

J.W. Campbell: Can you distinguish for an unovercoated echelle between the loss of efficiency due to scatter from the echelle profile and that due to oxidation of the aluminium film?

Have you measured the efficiency of unovercoated echelles as a function of time?

$B$. B. Jones: We have no data on the variation of the echelle efficiencies with time. With regard to the reflectivity of an aluminium oxide layer, this layer is formed almost immediately after the aluminium coating is completed and becomes approximately stable after about one year. Our gratings were well over one year old when measured and we have no information on any further change. 